\title{
Absceso pulmonar y empiema fulminante por Pseudomonas aeruginosa
}

\author{
Hsuan-Wei Chen, Jing-Quan Zheng y Te-Yu Lin, M.D.
}

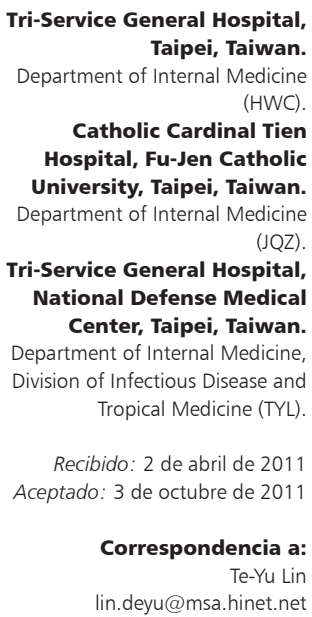

Tri-Service General Hospital, Taipei, Taiwan. Department of Internal Medicine (HWC). Catholic Cardinal Tien Hospital, Fu-Jen Catholic University, Taipei, Taiwan. Department of Internal Medicine (JQZ)

Tri-Service General Hospital, National Defense Medical Center, Taipei, Taiwan Department of Internal Medicine, Division of Infectious Disease and Tropical Medicine (TYL).

Recibido: 2 de abril de 2011 Aceptado: 3 de octubre de 2011

Correspondencia a: lin.deyu@msa.hinet.net

\section{Fulminant lobulated lung empyema caused by Pseudomonas aeruginosa}

We report the case of a 27-year-old man with a history of diabetes mellitus who presented with conscious disturbance, fever, and stiff neck after upper respiratory tract infection. Following diagnosis of meningoencephalitis, antibiotic therapy and deamethasone was initiated. He received endotracheal tube intervention under mechanical ventilation in the intensive care unit, and underwent successful weaning on day 4. One week later, he was diagnosed with pneumonia and a rapidly progressing lung empyema with abscess formation was noted. Microbiological culture of the pleural fluid revealed the presence of Pseudomonas aeruginosa. Nosocomial pneumonia is often caused by Staphylococcus aureus and $P$. aeruginosa; however, the latter often causes bronchopneumonia rather than fulminant empyema or lung abscess formation. The underlying diabetes mellitus and the history of steroid therapy may explain the present condition of this patient. The possibility of $P$. aeruginosa being the causative agent should be considered during differential diagnosis in patients presenting with fulminant lung empyema, especially in immunocompromised patients.

Key words: Fulminant lung empyema, Pseudomonas aeruginosa.

Palabras clave: Empiema pleural, Pseudomonas aeruginosa.

\section{Introducción}

$P$ seudomonas aeruginosa es un patógeno nosocomial común en pacientes con una enfermedad subyacente y colonizados en su vía respiratoria por esta bacteria, a la vez que dañada a consecuencia de la ventilación mecánica, traumas o virosis respiratorias recientes y una sobre respuesta inmune del hospedero. No obstante, infrecuentemente la neumonía por $P$. aeruginosa lleva a la formación de un absceso pulmonar aireado, en especial con un curso fulminante. Por otra parte, la etiología del empiema torácico ha cambiado, a raíz del empleo de antimicrobianos, desde una etiología estafilocóccica (Staphylococcus aureus) entre los años 1955 y 1965 a especies anaeróbicas estrictas ${ }^{1,2}$. Klebsiella pneumoniae es el patógeno más frecuentemente aislado en el empiema agudo y se asocia fuertemente con una diabetes mellitus subyacente. Presentamos un caso único en que una infección por $P$. aeruginosa condujo a la formación de un absceso pulmonar con curso fulminante en un paciente de 27 años, el que fue tratado exitosamente con drenaje y antibioterapia.

\section{Caso Clínico}

Un varón de 27 años, diabético, fue internado por fiebre, compromiso de conciencia y rigidez de nuca aparecidos tras tres días de cursar con una infección respiratoria aguda. Se le diagnosticó una meningoencefalitis aséptica (LCR de aspecto claro, con presión de $12 \mathrm{~cm} \mathrm{H}_{2} \mathrm{O}$; leucocitos: 200/mm³; tinción de Gram directa y cultivo bacteriano: negativos) y recibió ceftriaxona más vancomicina y corticoterapia (dexametasona, $10 \mathrm{mg}$ iv cada 6-horas durante 4 días). Inicialmente ingresó en una UCI y estuvo en ventilación mecánica, siendo desconectado a los 4 días.

Desarrolló fiebre y tos productiva tras una semana de evolución. Una Rx de tórax detectó un infiltrado pulmonar bilateral (Figura 1). Tres días más tarde, una segunda Rx reveló la presencia de múltiples abscesos pulmonares con niveles hidro-aéreos (Figura 2). La TAC de tórax comprobó la existencia de lesiones cavitarias (Figura 3) con formación de gas. Se le practicó una toracoscopia video-asistida e inserción de drenaje. Se inició entonces terapia asociada con linezolid (600 mg cada $12 \mathrm{~h} \mathrm{iv)} \mathrm{y} \mathrm{merope-}$ nem (1,0 gm cada $8 \mathrm{~h}$ iv), la que se mantuvo por dos semanas. La efusión pleural (de aspecto purulento, glucosa: $40 \mathrm{mg} / \mathrm{dL}, \mathrm{pH} 7,18$ ) dio un cultivo para $P$. aeruginosa (susceptible a amikacina, gentamicina, ciprofloxacina, cefepime, ceftazidima e imipenem). Hubo recuperación ad integrum luego de completarse el drenaje y la antibioterapia.

\section{Discusión}

Empiema pleural se define como la presencia de pus en el espacio pleural y es una complicación propia de la neumonía. Clínicamente se traduce con frecuencia en una grave inflamación y sepsis, requiriendo usualmente de drenaje y antibioterapia. Los pacientes con empiema pleural suelen tener fiebre, tos, dolor torácico, expectoración y disnea. En el caso presentado, el paciente contrajo una neumonía nosocomial y rápidamente evolucionó hacia la formación de un empiema y abscesos pulmonares causados por $P$. aeruginosa; a nuestro entender, este inusual comportamiento debiera haberse tenido presente por su rápida progresión hacia la complicación.

Pseudomonas aeruginosa no es causa común de infección pulmonar en hospederos previamente sanos, pero es un patógeno predominante en el caso de neumonías nosocomiales, especialmente en pacientes immunocomprometidos. En un estudio prospectivo, $P$. aeruginosa fue el bacilo gramnegativo más común en neumonías nosocomiales ${ }^{3}$ y una importante causa de traqueobronquitis intrahospitalaria ${ }^{4}$. En general, cuando $P$. aeruginosa es el único microorganismo aislado, la neumonía suele adquirir el carácter de bronconeumonía, no desarrollándose empiema ni abscesos pulmonares. 


\section{Comunicación Breve}

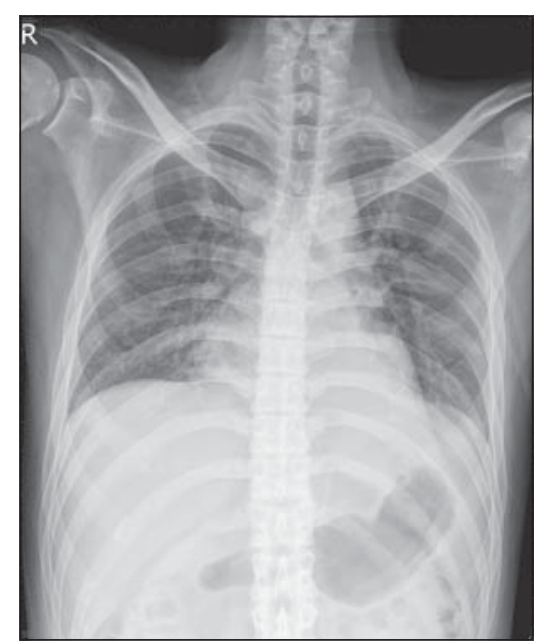

Figura 1. Radiografía de tórax que revela un infiltrado pulmonar bilateral.

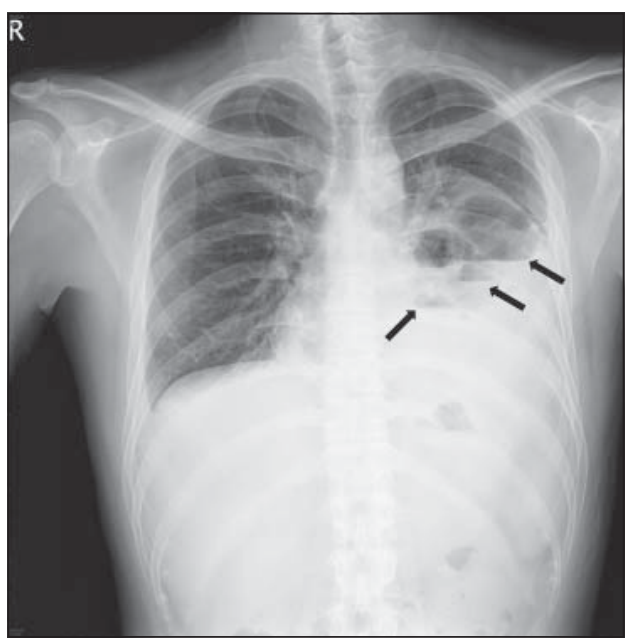

Figura 2. Radiografía de tórax: control tras 3 días. Revela múltiples lesiones con niveles hidro-aéreos (flecha).

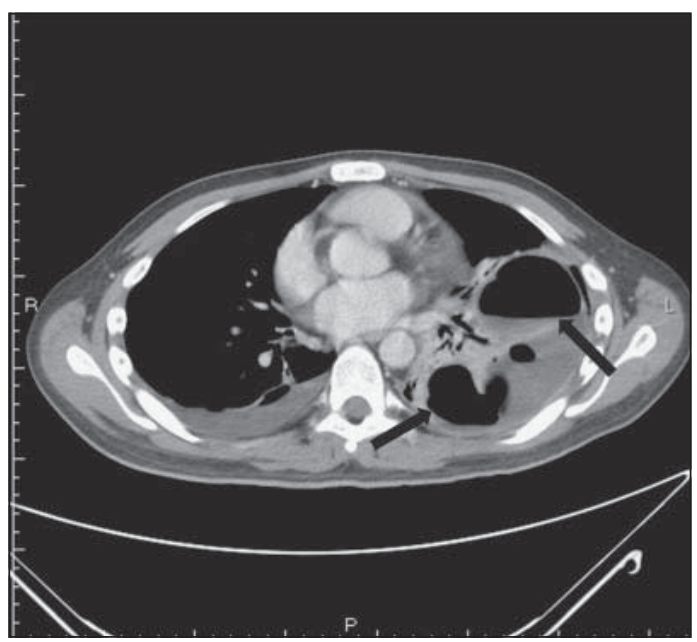

Figura 3. Tomografía axial de tórax. Revela la presencia de lesiones cavitarias (flecha) con formación de gas.
No obstante, Gluck et $\mathrm{al}^{5}$., comunicaron un caso en el cual un paciente contrajo una neumonía nosocomial por $P$. aeruginosa, evolucionando hacia la aparición de absceso pulmonar y empiema agudo.

Los hallazgos en la Rx de tórax son inespecíficos para esta etiología siendo la formación de cavidades y empiema extraordinariamente infrecuentes $^{6}$. Para diagnosticar la etiología por $P$. aeruginosa, son importantes los cultivos oportunos de líquido pleural, sangre y expectoración.

Los factores de riesgo para adquirir un empiema torácico agudo incluyen: diabetes mellitus, afecciones malignas, enfermedad pulmonar de base, enfermedad de los sistemas nervioso central o renal. La co-morbilidad más común es la diabetes mellitus, la que estuvo presente en nuestro paciente. Klebsiella pneumoniae es la etiología más común en esos casos.

Además de sus factores de virulencia, la flexibilidad genética de P. aeruginosa tiene también un rol en su supervivencia en diversos medioambientes; tiene un efecto destructivo directo sobre el parénquima pulmonar y sobre la respuesta inmune del hospedero ${ }^{8}$. En este caso, la formación fulminante de un empiema pulmonar podría estar condicionada por el antecedente de una corticoterapia reciente y la pre-existencia de diabetes mellitus.

Conclusión: El empiema pleural fulminante causado por P. aeruginosa es una entidad clínica inusual. Este caso nos refuerza que $P$. aeruginosa debiera ser una etiología a considerar en un paciente con empiema pleural rápidamente progresivo. Es importante la obtención oportuna de muestras de sangre, expectoración y líquido pleural para cultivo con el objeto de establecer la etiología. Tanto una antibioterapia adecuada como el drenaje exhaustivo son importantes para tener buen éxito en el tratamiento del empiema pleural por $P$. aeruginosa.

\section{Resumen}

Comunicamos el caso de un paciente del sexo masculino, con 27 años de edad, diabético que consultó por compromiso de conciencia, fiebre y rigidez cervical, después de haber cursado una infección respiratoria alta. Se diagnosticó una meningoencefalitis iniciándose antibioterapia y dexametasona. Fue atendido en UCI y conectado a ventilación mecánica siendo desconectado al cuarto día. Una semana más tarde, se le detectó una neumonía apreciándose la pronta instalación de un empiema pleural con formación de abscesos parenquimatosos. El cultivo del líquido pleural arrojó desarrollo de Pseudomonas aeruginosa.

La neumonía nosocomial suele ser causada por Staphylococcus aureus y $P$. aeruginosa; este segundo es causa de bronconeumonía más que de empiema fulminante y formación de absceso. La pre-existencia de diabetes mellitus y el antecedente de corticoterapia podría explicar la evolución de este paciente. Pseudomonas aeruginosa debiera ser considerada en el diagnóstico etiológico diferencial en pacientes con un absceso pulmonar fulminante, especialmente en hospederos inmunocomprometidos.

\section{Referencias}

1.- Finland M, Barne M W. Changing ecology of acute bacteria empyema: occurrence and mortality at Boston City Hospital during 12 selected years from 1935 to 1972. J Infect Dis 1978; 137: 274-91.

2.- Bartlett J G, Gorbach S L, Thadepalli H, Finegold S M. Bacteriology of empyema. Lancet 1974; 1: 338-40.

3.- $\quad$ Woske H J, Röding T, Schulz I, Lode H. Ventilator-associated pneumonia in a surgical intensive care unit: epidemiolgy, etiology and comparison of three bronchscopic methods for microbiological specimen sampling. Crit Care 2001; 5: 167-73.

4.- Garau J, Gómez L. Pseudomonas aeruginosa pneumonia. Curr Opin Infect Dis 2003; 16 (2): 135-43.

5.- Gluck MC, Levister EC, and Katz S. Pseudomonas abscess and empyema of the lung. Dis Chest 1968; 54 (4): 387-90.

6.- Winer-Muram H T, Jennings S G, Wunderink R G, Jones C B, Leeper K V Jr. Ventilator-associated Pseudomonas aeruginosa pneumonia: radiographic findings. Radiology 1995 Apr;195(1): 247-52.

7.- Chen K Y, Hsueh P R, Liaw Y S, Yang P C, Luh K T. A 10-year experience with bacteriology of acute thoracic empyema. Chest 2000; 117 (6): 1685-9.

8.- Sadikot R T, Blackwell T S, Christman J W, Prince A S. Pathogen-host interactions in Pseudomonas aeruginosa pneumonia. Am J Respir Crit Care Med 2005; 171 (11): 1209-23. 